Cardiology in the Young

cambridge.org/cty

\section{Brief Report}

Cite this article: Endo T, Ochiai D, and Tanaka M (2020) Right aortic arch with an aberrant left subclavian artery: sonographic features by HDlive Flow imaging. Cardiology in the Young 30: 892-893. doi: 10.1017/ S1047951120001079

Received: 7 February 2020

Accepted: 14 April 2020

First published online: 14 May 2020

\section{Keywords:}

Prenatal diagnosis; fetal echocardiography; CHD; vascular ring; HDlive Flow

\section{Author for correspondence:}

Daigo Ochiai, MD, PhD, Keio University School of Medicine, Shinanomachi, Shinjyuku-ku, Tokyo 160-8582, Japan. Tel: +81 33353 1211; Fax: +81332261667.

E-mail: ochiaidaigo@keio.jp (c) The Author(s), 2020. Published by Cambridge University Press. This is an Open Access article, distributed under the terms of the Creative Commons Attribution licence (http:// creativecommons.org/licenses/by/4.0/), which permits unrestricted re-use, distribution, and reproduction in any medium, provided the original work is properly cited.

\title{
Right aortic arch with an aberrant left subclavian artery: sonographic features by HDlive Flow imaging
}

\section{Toyohide Endo, Daigo Ochiai 10 and Mamoru Tanaka}

Department of Obstetrics and Gynecology, Keio University School of Medicine, Tokyo, Japan

\section{Abstract}

The right aortic arch is a congenital vascular anomaly, which may form a vascular ring. However, prenatal identification of the branching pattern of brachiocephalic vessels is often limited. In this paper, we clearly demonstrated the branching pattern of brachiocephalic vessels in a case of right aortic arch with an aberrant left subclavian artery using HDlive Flow imaging.

\section{Case Reports}

The right aortic arch is a congenital vascular anomaly, which may form a vascular ring that causes respiratory failure after birth. ${ }^{1-4}$ However, prenatal identification of the branching pattern of brachiocephalic vessels as shown by conventional two-dimensional echocardiography is often limited. Here, we clearly demonstrated the vascular arrangement in a fetus with right aortic arch with aberrant left subclavian artery using HDlive Flow imaging.

A 38 -year-old gravida 2 para 0 woman was referred to our hospital for a prenatal checkup. At 18 weeks of gestation, a fetal ultrasound screening demonstrated right aortic arch associated with the left ductus arteriosus (i.e. U shape sign), although no structural defects were noted except for the vascular abnormalities. At 26 weeks of gestation, fetal echocardiography using HDlive Flow imaging (Voluson E10; GE Healthcare, Zipf, Austria) demonstrated the following findings: the right aortic arch and the left ductus arteriosus consisted of a vascular ring surrounding the trachea (Figure 1a). The left common carotid artery, the right common carotid artery, and the right subclavian artery branched from the aortic arch in order. In addition, the left subclavian artery was arising near the junction of the aorta and the ductus arteriosus behind the trachea and the oesophagus (Figure 1b, Suppelementary Video S1, S2). Thus, the fetus was diagnosed with right aortic arch with the aberrant left subclavian artery. The female baby was delivered at 38 weeks of gestation, weighing $3111 \mathrm{~g}$ with Apgar scores of 5 and 8 at 1 and $5 \mathrm{~min}$, respectively. Her neonatal clinical course was uneventful except that the right aortic arch with the aberrant left subclavian artery was noted.

The various anomalies of right aortic arch with ductus arteriosus include right aortic arch with aberrant left subclavian artery; right aortic arch with mirror-image branching; double aortic arch; and isolation of left subclavian artery. Among them, right aortic arch with aberrant left subclavian artery and double aortic arch may form a vascular ring that causes respiratory failure. ${ }^{1-4}$ Therefore, a prenatal diagnosis of this vascular arrangement could contribute to optimal care after birth. However, prenatal identification of the branching pattern of brachiocephalic vessels is often limited by acoustic windows and sonographer experience. ${ }^{2}$ Recently, several clinical advantages of three-/ four-dimensional ultrasound imaging to diagnose fetal aortic arch anomalies have been reported. ${ }^{3-5}$ Regarding HDlive Flow imaging, once the volume data are acquired, we could manipulate the data to recreate views that enable identification of the vascular arrangement later. In the present case, all brachiocephalic vessels (i.e. left subclavian artery, left common carotid artery, right common carotid artery, and right subclavian artery) could be visualised on one screen at a glance (Figure 1 and Supplementary Video S1, S2). In addition, we could move the appropriate three-dimensional images in any direction to confirm the vascular arrangement (Supplementary Video S1, S2). Finally, glass body algorithms enabled us to demonstrate the tracheal lumen surrounded by the vascular ring by the adjustment of a light source (Figure 1a). Thus, HDlive Flow imaging could provide additional information about the fetus with aortic arch abnormalities like a vascular ring.

Supplementary material. To view supplementary material for this article, please visit https://doi.org/10.1017/ S1047951120001079

Acknowledgements. None.

Financial support. This research received no specific grant from any funding agency, commercial,f or not-for-profit sectors. 
(a)

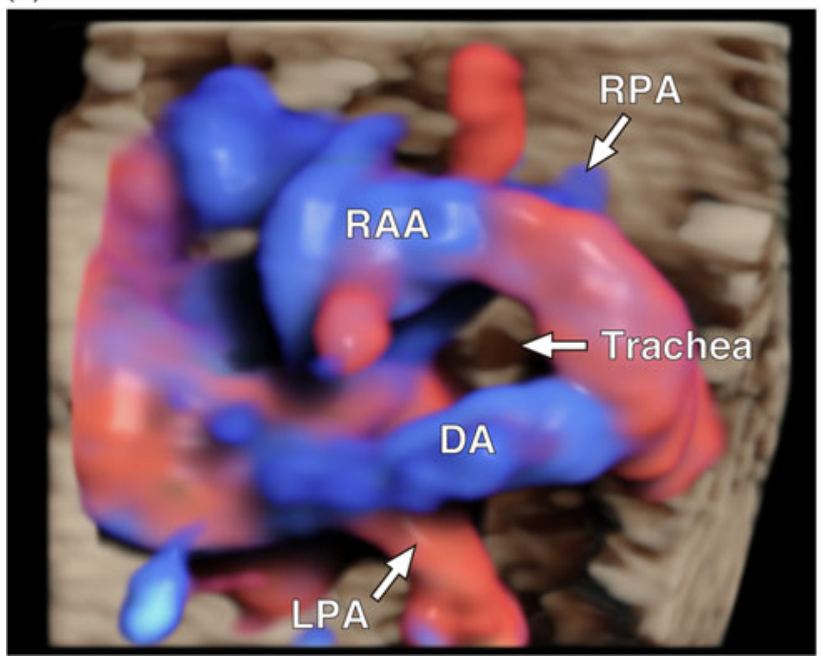

(b)

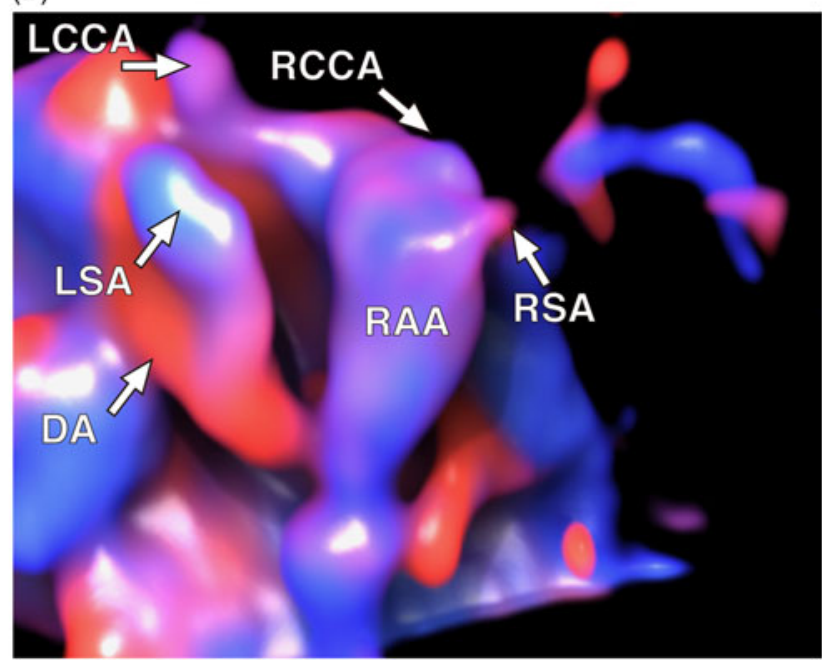

Figure 1. HDlive Flow imaging by glass body and rendering algorithms. (a) HDlive Flow imaging by glass body algorithms shows the vascular ring. Note that glass body mode clearly demonstrates the tracheal lumen. (b) HDlive Flow imaging by rendering algorithms shows the branching pattern of the brachiocephalic vessels. LCCA, left common carotid artery; RCCA, right common carotid artery; RSA, right subclavian artery; LSA, left subclavian artery; RAA, right aortic arch; DA, ductus arteriosus; RPA, right pulmonary artery; LPA, left pulmonary artery.

\section{Conflicts of interest. None.}

Ethical standards. All procedures followed were in accordance with the ethical standards of the responsible committee on human experimentation (institutional and national) and with the Helsinki Declaration of 1964 and later versions. Informed consent was obtained from the patient for publication of this case report.

\section{References}

1. Berg C, Bender F, Soukup M, et al. Right aortic arch detected in fetal life. Ultrasound Obstet Gynecol 2006;28:882-889.
2. Campanale CM, Pasquini L, Santangelo TP, et al. Prenatal echocardiographic assessment of right aortic arch. Ultrasound Obstet Gynecol 2019;54: 96-102.

3. Tseng JJ, Peng HW, Jan SL. An in-depth perspective of aortic arch branching in fetal vascular rings using spatiotemporal image correlation combined with high-definition flow imaging: report of 4 cases. J Ultrasound Med 2019;38: 2217-2224.

4. Yoo SJ, Min JY, Lee YH, Roman K, Jaeggi E, Smallhorn J. Fetal sonographic diagnosis of aortic arch anomalies. Ultrasound Obstet Gynecol 2003;22: 535-546.

5. Wang Y, Fan M, Siddiqui FA, et al. Strategies for accurate diagnosis of fetal aortic arch anomalies: benefits of three-dimensional sonography with spatiotemporal image correlation and a novel algorithm for volume analysis. J Am Soc Echocardiogr 2018;31:1238-1251. 